\title{
Genetic analysis of early onset familial coronary artery diseases
}

\author{
Mohammad Javad Ghorbani ${ }^{1,2}$, Nematollah Razmi ${ }^{3}$, Seyed Mohammad Bagher Tabei ${ }^{4}$, \\ Mohammad Javad Zibaeenezhad ${ }^{5}$, Hamid Reza Goodarzi²
}

\author{
${ }^{1}$ Department of Genetics, Fars Science and Research Branch, Islamic Azad University, \\ Marvdasht, Iran \\ ${ }^{2}$ Department of Genetics, Marvdasht Branch, Islamic Azad University, Marvdasht, Iran \\ ${ }^{3}$ Department of Biochemistry, Shiraz Branch, Islamic Azad University, Shiraz, Iran \\ ${ }^{4}$ Maternal-Fetal Medicine Research Center, Shiraz University of Medical Sciences, \\ Shiraz, Iran \\ ${ }^{5}$ Department of Cardiology, Shiraz University of Medical Sciences, Shiraz, Iran
}

Submitted: 1 August 2018

Accepted: 14 January 2019

Arch Med Sci Atheroscler Dis 2019; 4: e1-e6

DOI: https://doi.org/10.5114/amsad.2019.83149

Copyright (c 2019 Termedia \& Banach

\begin{abstract}
Introduction: Coronary artery diseases (CAD) are the most common causes of death. Myocardial infarction (MI) is a complex multifactorial and the most severe type of CAD. Early onset $\mathrm{MI}$ in a first-degree relative could be defined as an independent risk factor for CAD. This study was performed to investigate the genetic cause of early onset familial CAD.

Material and methods: In this study, the genetic cause of familial CAD was investigated in patients with a family history of CAD who underwent angiography before the age of 50 years. The patients did not have any diagnostic criteria for familial hypercholesterolemia, diabetes, or obesity, and also they were not opium or alcohol users. Whole exome sequencing in probands was performed and mutation was confirmed by PCR and Sanger sequencing.

Results: In our studied population, the c.501G>C (p.K167N) mutation in the $O L R 1$ gene was identified in a family. Mutation was confirmed by PCR and Sanger sequencing in the homozygous state (GG) in patients. Healthy individuals in this family were heterozygous (GC) and homozygous (CC).

Conclusions: This finding suggests that the $O L R 1$ gene could be a possible cause of early onset familial MI. Considering that parents of all affected individuals had a consanguineous marriage, it is important to perform carrier screening and genetic counseling in this family and their close relatives as a prevention strategy in populations at risk.
\end{abstract}

Key words: myocardial infarction, OLR1 gene, whole exome sequencing.

\section{Introduction}

According to the Third Report by the World Health Organization (WHO), 12 million people die annually of coronary artery diseases (CAD) worldwide [1]. In developing countries, the number of adults who develop CAD at a young age is high [2]. In Iran, CAD are the most common causes of death [1]. Recently, the Director of the Heart and Cardiovascular Center of Iran's Health Ministry emphasized that about 300 people die in Iran due to cardiovascular disease every day [3]. These diseases lead to complications, significant disability, and reduced productivity of patients

\author{
Corresponding author: \\ Nematollah Razmi \\ Shiraz Islamic \\ Azad University \\ Shiraz, Iran \\ Phone: +98 0713333324 \\ Fax: +98 0713333324 \\ E-mail: doctorrazmi@gmail. \\ com
}


[4]. The risk factors for CAD include age, gender, smoking, hypertension (HTN), hyperlipidemia, and diabetes, but are not limited to these factors [5]. Lifestyle factors are well-known risk factors for CAD but genetic factors also play an important role in the disease pathogenesis [6]. The American Heart Association reported in a recently update in 2017 that among adults $\geq 20$ years of age, $12.2 \%$ reported having a parent or sibling with a heart attack or angina before the age of 50 years [7]. These statistics indicate the importance of investigating the genetic cause of early onset familial CAD. The long-recognized familial clustering of CAD suggests that genetics plays a central role in its development, with the heritability of CAD estimated at approximately 50\% to 60\% [8]. Genetic investigations on early onset CAD patients can be more fruitful [9]. Early onset CAD $(<55$ in men and $<65$ in women) in a first-degree relative could be defined as an independent risk factor for CAD [8]. Whole exome sequencing (WES) is an exceptionally valuable screening tool for its capability to establish the clinical diagnosis of inherited CAD, particularly for poorly defined cases of sudden cardiac death [10].

Due to the importance of early onset familial $C A D$, this study was performed to investigate the genetic cause of early onset CAD.

\section{Material and methods}

In this study, 40 patients with premature CAD and myocardial infarction (MI) attending the Angiography Center of Namazi Hospital, Shiraz University of Medical Sciences were evaluated and referred for genetic counseling in Amin genetic counseling center, Marvdasht, Iran. The diagnosis of MI was referred to the Third Universal Definition of Myocardial Infarction [11] and patients were identified as having definite $\mathrm{MI}$ and premature CAD on the basis of coronary angiography. We selected the familial form of premature CAD and $\mathrm{MI}$ (at least 5 patients in a pedigree) and patients that did not have known risk factors for cardiovascular disease to increase the chances of finding effective genetic factors in the incidence of $\mathrm{MI}$ and premature CAD. Information about age, gender, family history of CAD (male and female first-degree relatives $<50$ years old), dyslipidemia (high low-density lipoprotein (LDL) cholesterol based on ATP III or high-density lipoprotein (HDL) cholesterol < $40 \mathrm{mg} / \mathrm{dl}$ or triglycerides > $150 \mathrm{mg}$ / dl) [12], diabetes mellitus (fasting blood glucose $\geq 126 \mathrm{mg} / \mathrm{dl}, 2 \mathrm{~h}$ postprandial glucose $\geq 200 \mathrm{mg} / \mathrm{dl}$, or use of hypoglycemic agents or insulin), hypertension (positive past history of hypertension or use of antihypertensive drugs), smoking, and opium consumption were collected from all patient's medical record and the coronary angiography report including the deceased patients. Patients and families who were diagnosed with dyslipidemia, diabetes mellitus, smoking or opium consumption were excluded from the study. After informed consent, 5-6 $\mathrm{ml}$ blood samples were taken from patients. Genomic DNA was extracted from peripheral blood samples using a High Pure PCR Template Preparation Kit (Roche Life Science, Germany). After qualitative and quantitative assessment using standard techniques, DNA was subjected to WES. We used the homozygosity strategy for consan-

Table I. Sequence of forward and reverse PCR primers

\begin{tabular}{|cc|}
\hline Forward & Reverse \\
\hline TCCTTGTCCGCAAGACTGGAT & GGCATCAAAGGAGAACCGTC \\
\hline
\end{tabular}

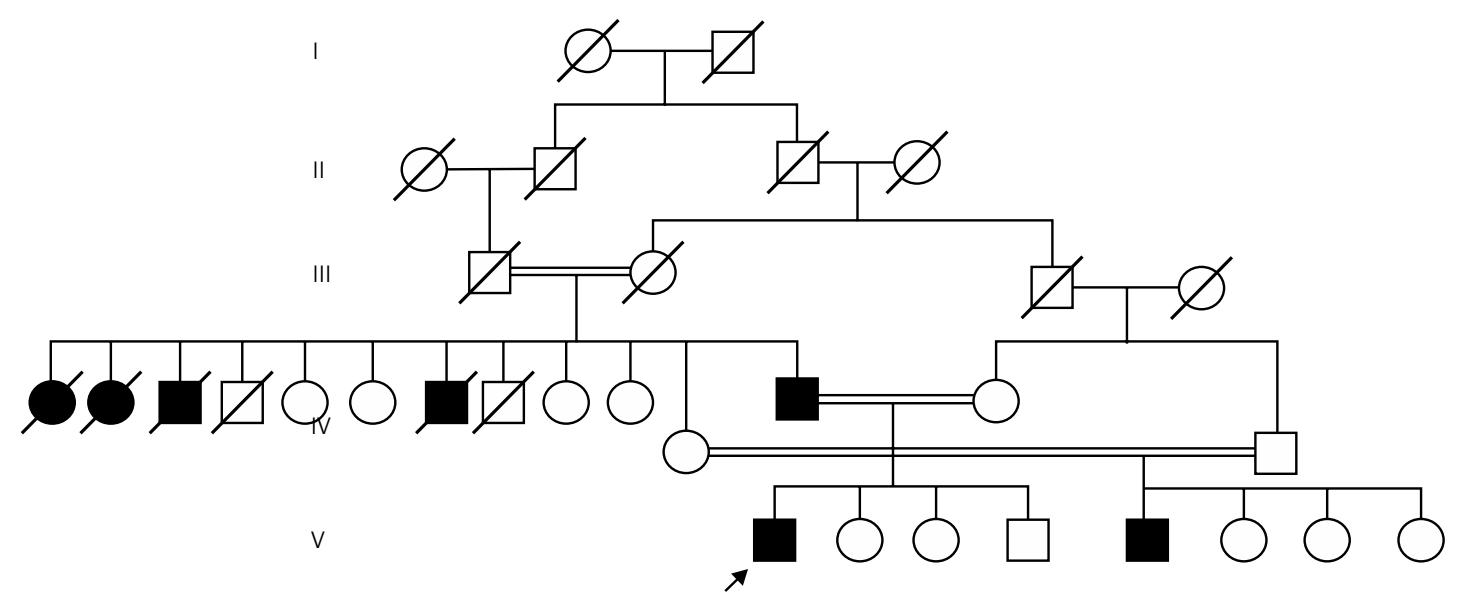

Figure 1. Pedigree of a family demonstrating autosomal recessive inheritance of early onset MI. Individuals with early onset MI are indicated by solid squares (males) or solid circles (females). Unaffected individuals are indicated by open symbols. Deceased individuals are indicated by a slash (/). The proband is indicated by an arrow 
guineous pedigrees [13]. Considering that parents of all affected individuals had a consanguineous marriage, the pedigree suggested homozygous recessive mode of inheritance. We performed sequencing of a single affected person to identify a homozygous mutation in a gene located within a homozygous region. Paired-end 101 base pair sequencing was performed on Illumina's HiSeq2000 platform (Illumina, San Diego, CA, USA). Whole exome sequencing was performed in patients. In WES reports, 51180 variants were identified. Variants were first filtered based on quality criteria. Subsequently, variants outside the coding regions were filtered out, as well as synony-

Proband ( $\mathrm{R}$ primer)

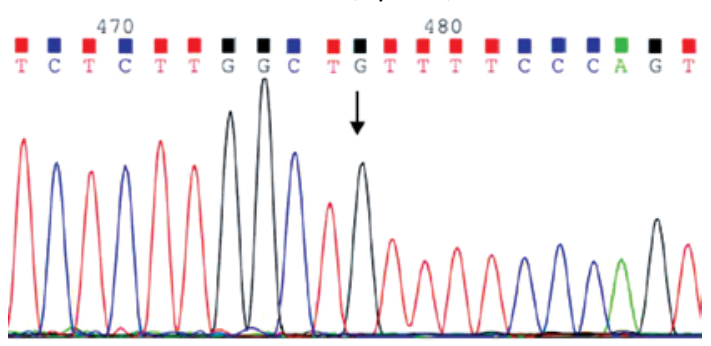

IV-12 (R primer)
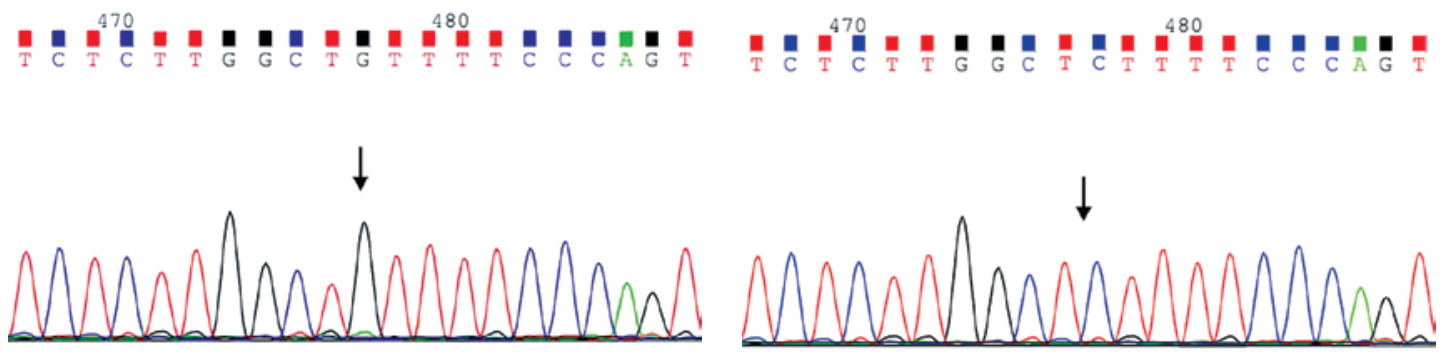

mous coding variants. After analysis, we identified a suspected mutation. The c.501G>C (p.K167N) missense mutation was identified in the OLR1 gene, in the homozygous state in a proband by WES, and PCR primers (Table I) were designed manually and using primer 3 and Primer-BLAST to amplify the mutations containing fragments. PCR amplification was carried out in a total volume of $25 \mu \mathrm{l}$ containing $30 \mathrm{ng}$ of genomic DNA, $12.5 \mu \mathrm{l}$ PCR Master Mix (Promega, Madison, USA), $1 \mu$ of each primer and $5.5 \mu \mathrm{l}$ of DDW. The thermal profile included initial denaturation at $95^{\circ} \mathrm{C}$ for $5 \mathrm{~min}$, followed by 34 cycles of denaturation at $95^{\circ} \mathrm{C}$ for $15 \mathrm{~s}$, annealing and extension at $60^{\circ} \mathrm{C}$ for $60 \mathrm{~s}$,

V-05 (R primer)
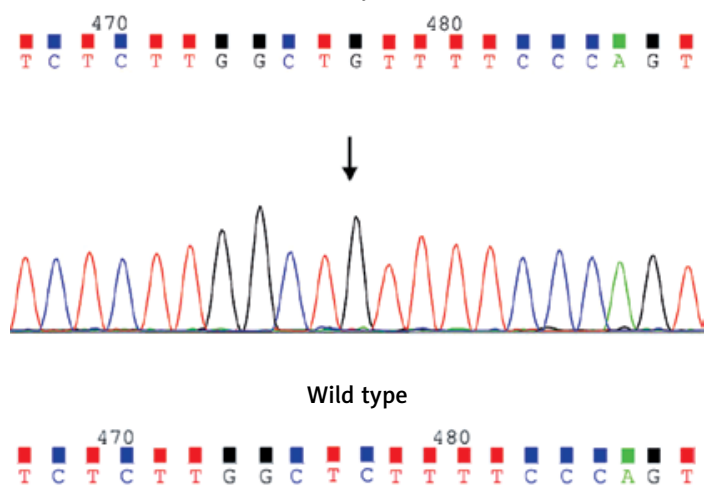
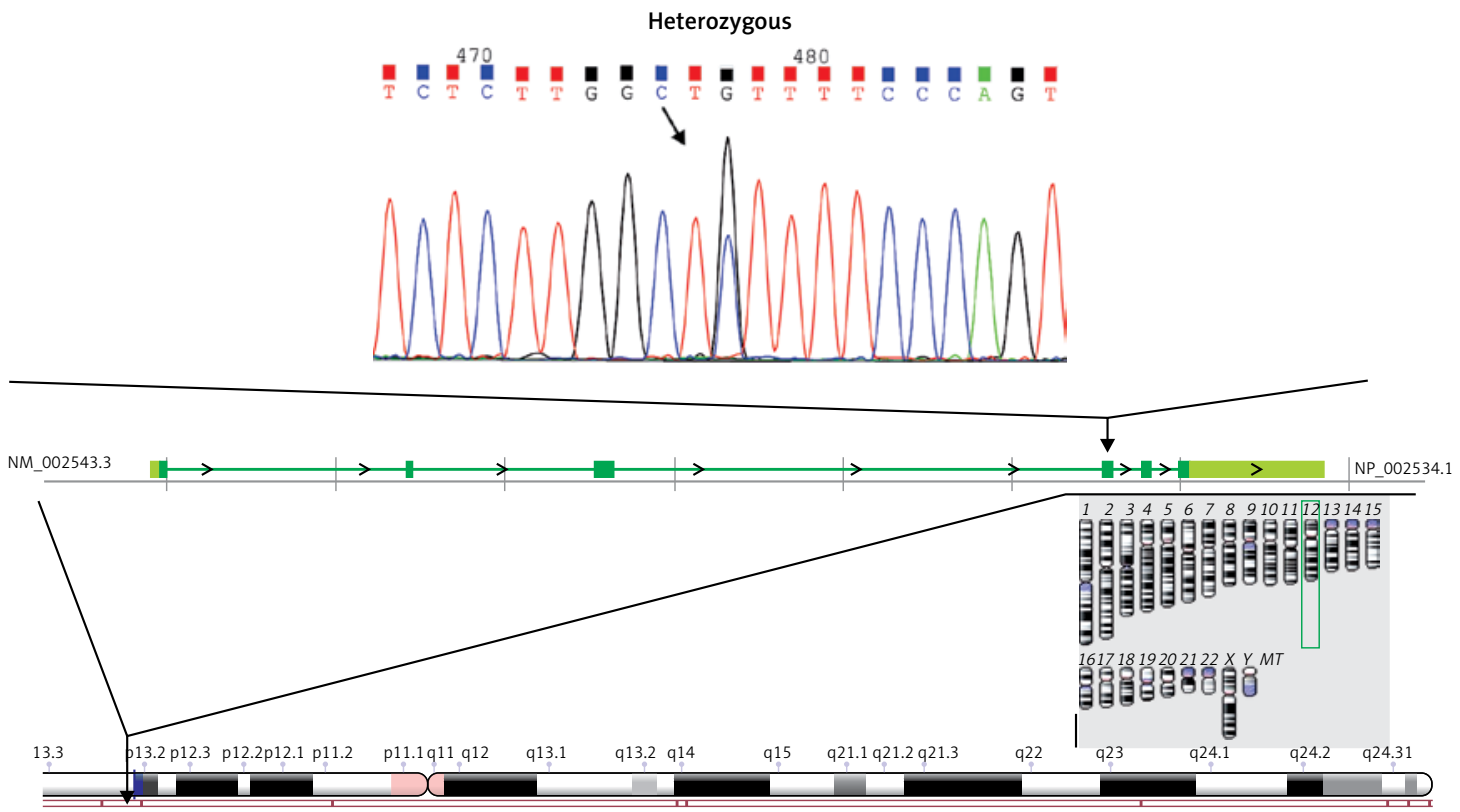

Figure 2. Truncated sequencing chromatogram of $O L R 1$ gene of patients. The mutation point is indicated by an arrow 


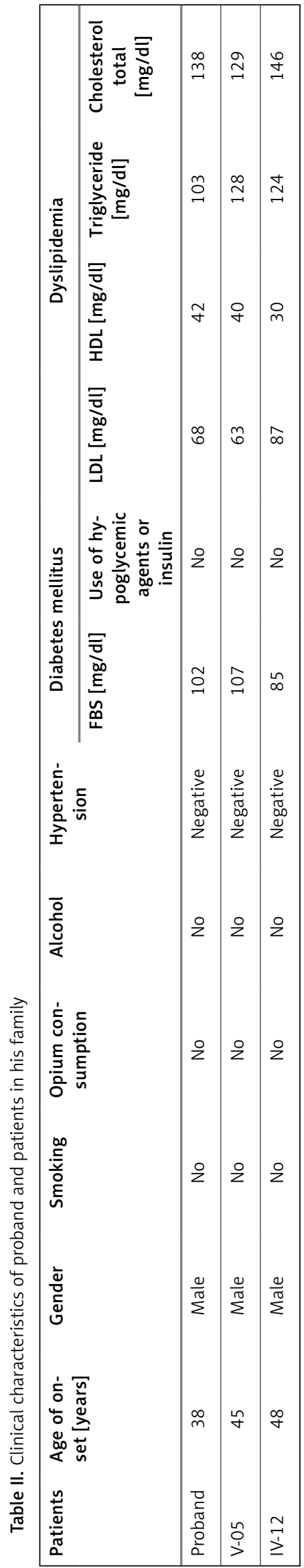

and a final extension at $72^{\circ} \mathrm{C}$ for 10 min. Sanger sequencing was performed to confirm mutations in probands and their family members.

\section{Results}

The present study identified an Iranian family containing 32 members (16 members deceased) with a history of early onset $\mathrm{MI}$ and premature CAD (Figure 1). The family consisted of 18 females and 14 males distributed in five generations; seven members were diagnosed with premature CAD and MI (four of whom were deceased). The proband (V.1) with two-vessel disease of the left anterior descending (LAD) and right coronary artery (RCA) was identified at the Department of Cardiology, Namazi Hospital, Shiraz University of Medical Sciences at 38 years of age. Subsequently, the patient's father, paternal uncles and aunts, and his male cousin developed symptoms of MI. As shown in Table II, a few members of the family (V.4 and IV.11) had hypertension, but all had normal serum levels of low-density and high-density lipoproteins and serum levels of total cholesterol and triglycerides, and none of the family members were cigarette smokers or had hypertension, diabetes or obesity. These clinical manifestations strongly suggested heritable CAD in this family. Pedigree analysis of the family suggested autosomal recessive inheritance of CAD (Figure $1 \mathrm{~A}$ ). The c.501G >C (p.K167N) missense mutation was identified in the OLR1 gene in the homozygous state in patients by WES. Although WES is a method of high throughput sequencing, it has not been approved for clinical and diagnostic use; therefore mutation was confirmed by PCR and Sanger sequencing (Figure 2). There are no data about the first, second and third generation. Segregation analysis revealed that V.5's parents and IV.13 were heterozygous. Also, all the proband's siblings and one of V.5's sisters were heterozygous.

\section{Discussion}

The OLR1 gene encodes a cell-surface endocytosis receptor for oxidized low-density lipoprotein (OxLDL) which is termed LOX1. LOX1 is expressed on vascular smooth muscle cells and the plasma membrane of differentiated macrophages [14]. Hypoxia increases lipid uptake into macrophages and differentially regulates the expression of oxLDL receptors. Lox-1 plays a major role in hypoxia-induced foam cell formation [15]. We found a family in our studied population with missense mutation c.501G>C (p.K167N) in the OLR1 gene in the homozygous state. The $\mathrm{K} 167 \mathrm{~N}$ polymorphism causes an amino acid substitution at codon 167 (Lys to Asn) which is located within the C-terminal domain of the protein. Replacement of this Lys residue causes reduced binding and internal- 
ization of oxLDL [16]. The association reported in the present study confirms the previously reported association of the OLR1 gene product in the pathogenesis of MI. Tatsuguchi et al. reported a positive association of the $\mathrm{K} 167 \mathrm{~N}$ polymorphism with the risk of myocardial infarction in a sample of Japanese patients [17]. Wang et al. reported that the p.K167N missense mutation in the OLR1 gene causes plaque formation and Predazzi et al. studied a population that included 2141 patients and concluded that the p.K167N missense mutation in the OLR1 gene causes plaque formation in the carotid arteries [18, 19]. However, Sakowicz et al. and Trabetti et al. cannot find any relation between $\mathrm{G} 501 \mathrm{C}$ genotype and acute myocardial infarction $[14,20]$. This discrepancy might be due to differences in the genetic background between populations.

In conclusion, in the work-up of patients, we found that patients have hypertension before the age of onset and control it after MI by use of antihypertensive drugs. LOX1 plays diverse roles in cardiovascular diseases, such as atherosclerosis and hypertension. The basal expression of LOX-1 is low, but can be enhanced by hypertension [21]. Hou et al. showed that polymorphisms at G501C in the LOX-1 gene is associated with susceptibility to hypertension [22]. They studied 280 patients with hypertension and 284 control subjects in China. They reported that the CC genotype of OLR-1 G501C polymorphism is associated with susceptibility to hypertension in the Chinese population. To the best of our knowledge, this is the first report to identify an OLR1 mutation in a family with premature CAD. In this study, we found seven patients with $\mathrm{MI}$ among whom four patients died before the age of 50 years. Considering that parents of all affected individuals had consanguineous marriages, it is important to perform carrier screening and genetic counseling in this family and their close relatives as a prevention strategy in populations at risk. The findings in this report show that mutations in the OLR1 gene could possibly be a common cause of early onset familial CAD and $M I$ in patients.

\section{Acknowledgments}

Special sincere thanks to all the patients and their families participated in this research.

\section{Conflict of interest}

The authors declare no conflict of interest.

\section{References}

1. Mohseni J, Kazemi T, Maleki MH, Beydokhti H. A systematic review on the prevalence of acute myocardial infarction in Iran. Heart Views 2017; 18: 125-32.
2. Abed MA, Eshah NF, Moser DK. Risk profile of myocardial infarction in young versus older adults. Heart Lung 2018; 47: 226-30.

3. Biglu MH, Ghavami M, Biglu S. Cardiovascular diseases in the mirror of science. J Cardiovasc Thorac Res 2016; 8: 158-63.

4. Firoozabadi MD, Kazemi T. A memorandum of "World Heart Day 2013" - Stroke mortality among women in Birjand, East of Iran. Iran J Nurs Midwifery Res 2014; 19: 215.

5. Chen QF, Wang W, Huang Z, et al. Correlation of rs1122608 SNP with acute myocardial infarction susceptibility and clinical characteristics in a Chinese Han population: a case-control study. Anatol J Cardiol 2018; 19: 249-58.

6. Kelloniemi A, Szabo Z, Serpi R, et al. The early-onset myocardial infarction associated phactr1 gene regulates skeletal and cardiac alpha-actin gene expression. PLoS One 2015; 10: e0130502.

7. Benjamin EJ, Blaha MJ, Chiuve SE, et al. Heart disease and stroke statistics-2017 update: a report from the American Heart Association. Circulation 2017; 135: e146-603.

8. Dai X, Wiernek S, Evans JP, Runge MS. Genetics of coronary artery disease and myocardial infarction. World J Cardiol 2016; 8: 1-23.

9. InanlooRahatloo K, Parsa AF, Huse K, et al. Mutation in ST6GALNAC5 identified in family with coronary artery disease. Sci Rep 2014; 4: 3595.

10. Seidelmann SB, Smith E, Subrahmanyan L, et al. Application of whole exome sequencing in the clinical diagnosis and management of inherited cardiovascular diseases in adults. Circ Cardiovasc Genet 2017; 10: pii: e001573.

11. Thygesen K, Alpert JS, Jaffe AS, et al. Third universal definition of myocardial infarction. J Am Coll Cardiol 2012; 60: 1581-98.

12. National Cholesterol Education Program (NCEP) Expert Panel on Detection, Evaluation, and Treatment of High Blood Cholesterol in Adults (Adult Treatment Panel III). Third Report of the National Cholesterol Education Program (NCEP) Expert Panel on Detection, Evaluation, and Treatment of High Blood Cholesterol in Adults (Adult Treatment Panel III) final report. Circulation 2002; 106: 3143-421.

13. Gilissen C, Hoischen A, Brunner HG, Veltman JA. Disease gene identification strategies for exome sequencing. Eur J Hum Genet 2012; 20: 490-7.

14. Sakowicz A, Fendler W, Lelonek M, Pietrucha T. Genetic variability and the risk of myocardial infarction in Poles under 45 years of age. Arch Med Sci 2010; 6: 160-7.

15. Crucet M, Wüst SJ, Spielmann P, Lüscher TF, Wenger RH, Matter CM. Hypoxia enhances lipid uptake in macrophages: role of the scavenger receptors Lox1, SRA, and CD36. Atherosclerosis 2013; 229: 110-7.

16. Chen M, Narumiya S, Masaki T, Sawamura T. Conserved C-terminal residues within the lectin-like domain of LOX-1 are essential for oxidized low-density-lipoprotein binding. Biochem J 2001; 355: 289-96.

17. Tatsuguchi M, Furutani M, Hinagata J, et al. Oxidized LDL receptor gene (OLR1) is associated with the risk of myocardial infarction. Biochem Biophys Res Commun 2003; 303: 247-50.

18. Wang L, Yanuck D, Beecham A, et al. Candidate gene study revealed sex-specific association between the OLR1 gene and carotid plaque. Stroke 2011; 42: 588-92.

19. Predazzi IM, Norata GD, Vecchione L, et al. Association between OLR1 K167N SNP and intima media thickness of the common carotid artery in the general population. PLoS One 2012; 7: e31086. 
20. Trabetti E, Biscuola M, Cavallari U, et al. On the association of the oxidised LDL receptor 1 (OLR1) gene in patients with acute myocardial infarction or coronary artery disease. Eur J Hum Genet 2006; 14: 127-30.

21. Ogura S, Kakino A, Sato Y, et al. Lox-1: the multifunctional receptor underlying cardiovascular dysfunction. Circ J 2009; 73: 1993-9.

22. Hou XW, Wang LF, Wang N, et al. The G501C polymorphism of oxidized LDL receptor gene [OLR-1] is associated with susceptibility and serum C-reactive protein concentration in Chinese essential hypertensives. Clin Chim Acta 2008; 388: 200-3. 